\title{
Quasicontinuum modeling of short-wave instabilities in crystal lattices
}

\author{
LEV TRUSKINOVSKY ${ }^{\dagger}$ \\ Laboratoire de Mechanique des Solides, CNRS-UMR 7649, Ecole Polytechnique, \\ Palaiseau, 91128, France \\ and ANNA VAINCHTEIN $\ddagger$ \\ Department of Mathematics, University of Pittsburgh, Pittsburgh, PA 15260, USA
}

We propose a hybrid quasicontinuum model which captures both long and short-wave instabilities of crystal lattices and combines the advantages of weakly nonlocal (higher gradient) and strongly nonlocal (integral) continuum models. To illustrate the idea, we study the simplest onedimensional lattice exhibiting commensurate and incommensurate short-wave instabilities. We explicitly compute stability limits of the homogeneous states using both discrete and quasicontinuum models. The new quasicontinuum approximation is shown to be capable of reproducing a detailed structure of the discrete stability diagram.

Keywords: Crystal lattice; Short-wave instability; Quasicontinuum model

\section{Introduction}

Lattice instabilities are responsible for various transformations of crystal structures. Macroscopic or long-wave instabilities give rise to martensitic phase transformations, which proceed through the formation of finite-size domains of the new phase [1, 2]. Microscopic or short-wave instabilities lead to the formation of multi-lattices and modulated "tweed" patterns $[2,3,4]$. Both micro and macroinstabilities are detectable in the phonon dispersion spectra. The loss of stability takes place when when the minimum of the dispersion curve touches the zero frequency level $[5,6,7]$. Macroscopic instabilities correspond to infinitely long waves and can be linked to the softening of the appropriate combinations of macroscopic elastic moduli. Typical microscopic instabilities occur at finite wave lengths associated with special points in the Brillouin zone corresponding to modulations commensurate with the lattice. Less frequent microinstabilities with the generic wave vectors give rise to incommensurate phases [8].

Classical continuum description of lattice instabilities is provided by the Landau theory. When the unstable wave vector is equal to zero, the order parameter can be chosen to coincide with a component of the macroscopic strain (e.g. [9]). If the unstable wave vector

\footnotetext{
$\dagger$ Email: trusk@lms.polytechnique.fr

${ }^{\ddagger}$ Corresponding author. Email: aav4@pitt.edu
} 
is different from zero, the macroscopic order parameter can be identified with the amplitude of an unstable normal mode (e.g. [10]). The resulting coarse-grained description remains adequate until external length scales become comparable with the lattice spacing as in the cases of ultra-thin layers, boundaries with singularities or strongly interacting defects.

To preserve the fine structure of the elastic fields in the continuum setting we propose a new quasicontinuum model which captures both long and short-wave instabilities associated with the acoustic branch of the phonon spectrum. The model is obtained by a long-wave expansion of the lattice energy and it shares with the discrete model the property that strain is the only order parameter. To make the truncated gradient expansion well-posed, we extend the polynomial dispersion relation by zero outside the first Brillouin zone. The model therefore restricts modulations in the physical space to length scales larger than lattice spacing. The suppression of the gradient model outside the Brillouin zone allows one to avoid the unphysical small-scale instabilities; a similar hard cutoff procedure is routinely used in coarse-grained continuum field theories [11]. As we show, the cutoff makes the gradient model equivalent to a fully nonlocal model with a slowly decaying oscillatory kernel.

To illustrate the idea, we consider a one-dimensional lattice involving interactions of up to third nearest neighbors which can be either of ferromagnetic or antiferromagnetic type. The discrete model is shown to generate both commensurate and incommensurate short-wave instabilities (e.g. $[12,13]$ ). We show that the quasicontinuum model captures all unstable modes exhibited by the discrete model and provides good quantitative bounds for the instability thresholds.

\section{Discrete model}

Consider an infinite chain of interacting particles with the total energy

$$
W=\varepsilon \sum_{n=-\infty}^{\infty} \sum_{p=1}^{q} p \phi_{p}\left(\frac{u_{n+p}-u_{n}}{p \varepsilon}\right) .
$$

Here $\phi_{p}(w)$ is the energy density of an effective spring with reference length $p \varepsilon$ (representing interaction of $p$ th nearest neighbors) and $u_{n}(t)$ is the displacement of $n$th particle. The equilibria in this system satisfy the following infinite system of difference equations:

$$
\sum_{p=1}^{q}\left[\phi_{p}^{\prime}\left(\frac{u_{n+p}-u_{n}}{p \varepsilon}\right)-\phi_{p}^{\prime}\left(\frac{u_{n}-u_{n-p}}{p \varepsilon}\right)\right]=0 .
$$

To access stability of a homogeneous equilibrium state $u_{n}^{0}=n \varepsilon w$, where $w$ is the average strain, we need to introduce perturbations $v_{n}=u_{n}-u_{n}^{0}$ and study the positive 
definiteness of the quadratic part of the energy expansion. This leads to the following eigenvalue problem:

$$
-\omega^{2} v_{n}=\sum_{p=1}^{q} K_{p}\left(v_{n+p}-2 v_{n}+v_{n-p}\right),
$$

where $K_{p}=\phi_{p}^{\prime \prime}(w) / p$ and $\omega^{2}$ is the square of the characteristic frequency. By representing the normal modes (phonons) in the form $v_{n}=\exp (i n k)$, where $k$ is a real wave number, we obtain the dispersion relation

$$
\omega^{2}(k)=4 \sum_{p=1}^{q} K_{p} \sin ^{2} \frac{p k}{2} .
$$

By symmetry and periodicity it suffices to consider the wave numbers in the interval $0 \leq k \leq \pi$ (Brillouin zone). A uniform deformation is then stable if and only if $\omega^{2}(k)>0$ for all $k \in(0, \pi]$. This condition is the far reaching generalization of the LegendreHadamard condition of strong ellipticity in continuum elasticity [14].

One can obtain some immediate necessary conditions for stability by requiring that

$$
\frac{d^{2} \omega^{2}(0)}{d k^{2}}>0 \text { and } \omega^{2}(\pi)>0
$$

The first condition, written as

$$
E=\sum_{p=1}^{q} p^{2} K_{p}>0
$$

means physically that the effective elastic modulus along the homogeneous branch of equilibria is positive; the corresponding eigenmode, $v_{n}=1$, is infinitely long wave. The second condition,

$$
\sum_{k=1, k \text { odd }}^{q} K_{p}>0
$$

is less transparent: the corresponding unstable eigenmode $v_{n}=(-1)^{n}$ is microscopic, commensurate and has the smallest possible wave length.

To characterize the complete set of stability conditions (necessary and sufficient) explicitly, we need to specify the number of interactions. One can show that the first generic case is $q=3$, when nearest (NN), next to nearest (NNN) and next to next to nearest (NNNN) neighbors interact. The corresponding dispersion relation reads

$$
\omega^{2}(k)=4 \sin ^{2} \frac{k}{2}\left(K_{1}+4 K_{2}+9 K_{3}-4\left(K_{2}+6 K_{3}\right) \sin ^{2} \frac{k}{2}+16 K_{3} \sin ^{4} \frac{k}{2}\right)
$$


A straightforward analysis of the positive definiteness of the right hand side produces the following necessary and sufficient conditions of stability:

$$
\begin{aligned}
& K_{1}+4 K_{2}+9 K_{3}>0 \\
& K_{1}+K_{3}>0 \\
& K_{2}^{2}<4 K_{3}\left(K_{1}+K_{2}\right) \text { if }-6<\frac{K_{2}}{K_{3}}<2
\end{aligned}
$$

The first two of these conditions have been already obtained as necessary (see (5), (6)) and can be identified with macroinstabilities $(k=0)$ and commensurate microinstabilities $(k=\pi)$, respectively. The third condition $(8)_{3}$ is associated with the incommensurate mode $0<k=2 \arcsin \sqrt{\left(K_{2}+6 K_{3}\right) /\left(8 K_{3}\right)}<\pi$. The full stability diagram in the plane

$$
K_{1}>0
$$

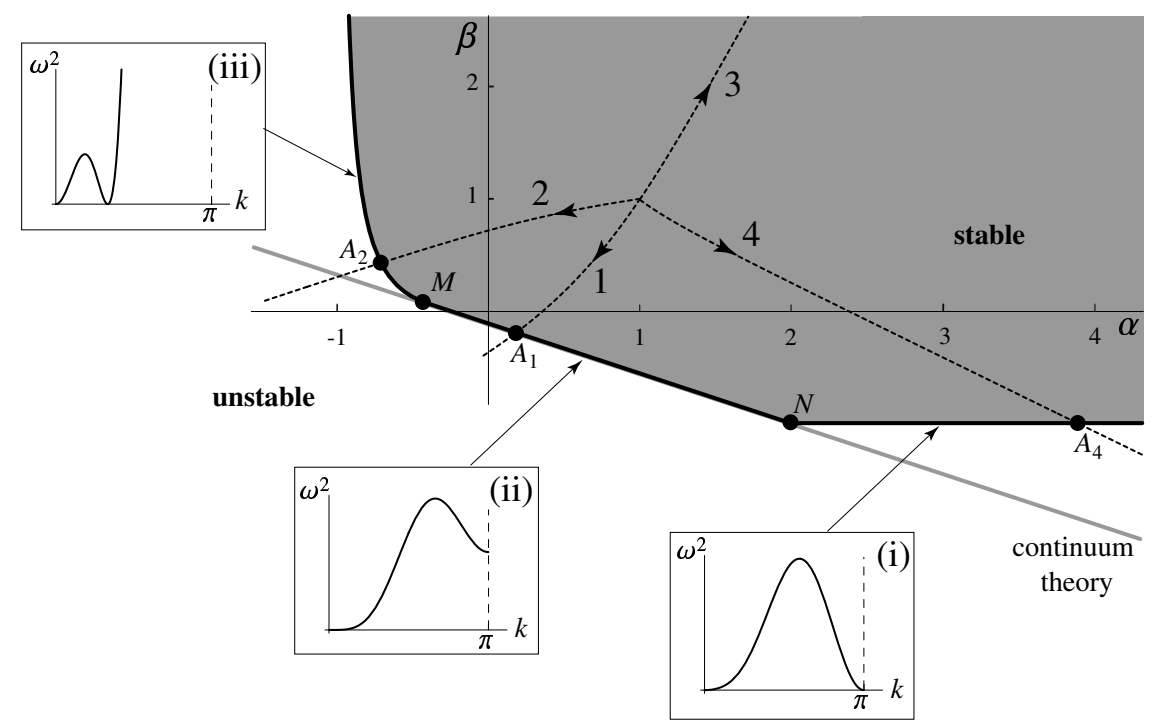

Figure 1: Stability diagram for the discrete model with $q=3$ and $K_{1}>0$. Regions of stability are shown in gray. Dispersion relations corresponding to different modes of stability loss are shown in inserts. Stability boundaries: (i) commensurate microinstability, $\beta=-1$ (to the right of $N$ ); (ii) macroinstability, $1+4 \alpha+9 \beta=0$ (between $N$ and $M$ ); (iii) incommensurate microinstability, $\alpha^{2}=4 \beta(1+\alpha)$ (above $M$ and below $R$ ). Classical continuum stability boundary is given by a gray line, $1+4 \alpha+9 \beta=0$. Dashed lines with numbers indicate deformation paths: $\gamma_{2}=\gamma_{3}=1$ (path 1); $\gamma_{2}=1, \gamma_{3}=0.4$ (path 2); $\gamma_{2}=0.4, \gamma_{3}=0.2($ path 3$) ; \gamma_{2}=\gamma_{3}=0.4$ (path 4). In all cases $\mu_{1}=\mu_{2}=\mu_{3}=\gamma_{1}=1$.

of the nondimensional parameters

$$
\alpha=\frac{K_{2}}{K_{1}}, \quad \beta=\frac{K_{3}}{K_{1}}
$$


$K_{1}<0$

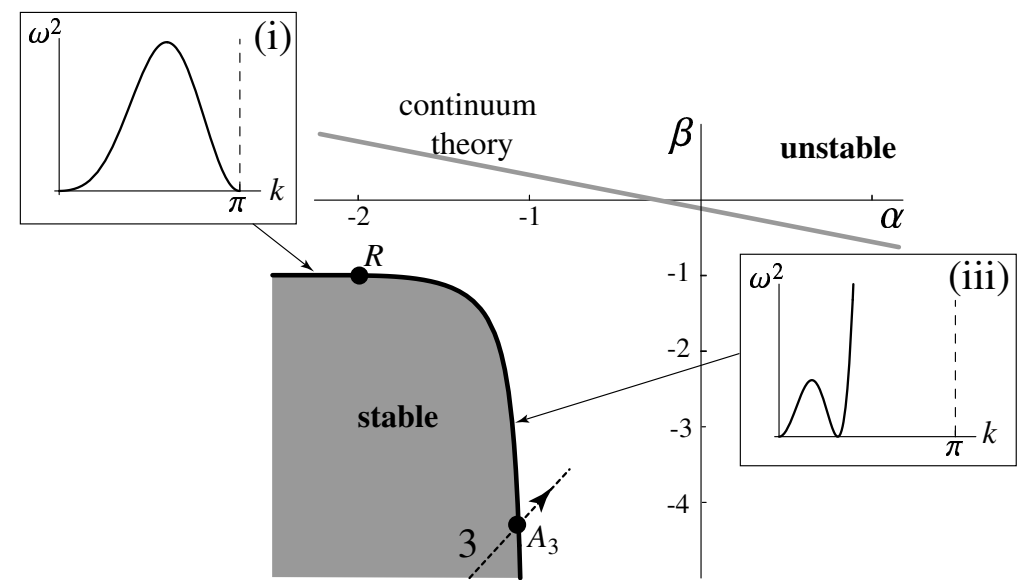

Figure 2: Stability diagram for the discrete model with $q=3$ and $K_{1}<0$. Stability boundaries: (i) commensurate microinstability, $\beta=-1$ (to the left of $R$ ); (iii) incommensurate microinstability, $\alpha^{2}=4 \beta(1+\alpha)$ (below $R$ ). Dashed line indicates deformation path 3 which is also shown in figure 1.

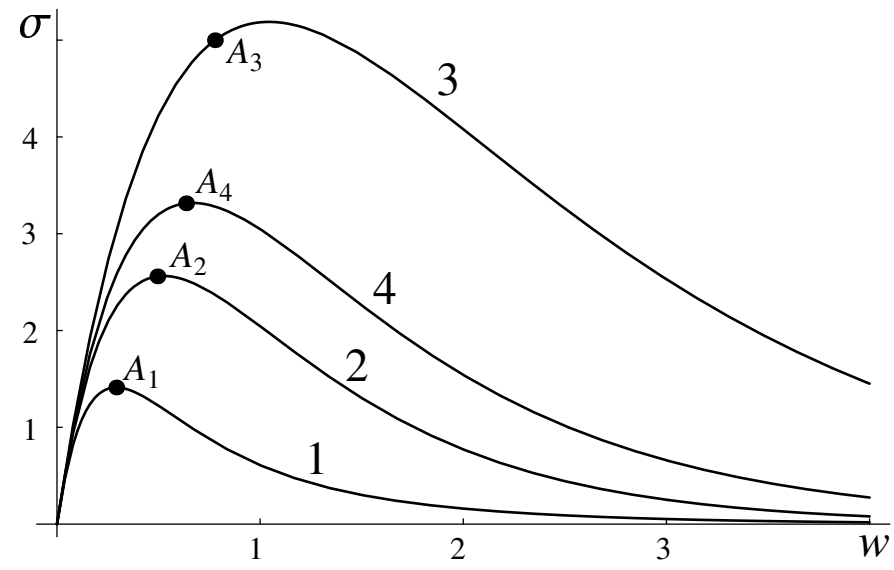

Figure 3: Stress-strain relations along the deformation paths shown in figures 1 and 2. Points $A_{i}$ mark the loss of stability of the homogeneous deformation. 
is presented in figures 1 for $K_{1}>0$ and 2 for $K_{1}<0$.

To relate the microscopic stability limits with the macroscopic stress-strain relation, consider a family of homogeneous configurations $u_{n}=w n \varepsilon$ parametrized by the strain $w$. Assume that all three interactions (NN, NNN and NNNN) are governed by the Morse potentials

$$
U_{p}(r)=\frac{\mu_{p} \varepsilon}{2 \gamma_{p}^{2}}\left(1-\exp \left[-\gamma_{p}\left(\frac{r}{\varepsilon}-1\right)\right]\right)^{2}, \quad 1 \leq p \leq 3 .
$$

The energy density associated with $p$ th interaction in the homogeneous state is

$$
\phi_{p}(w)=U_{p}(p \varepsilon(w+1)) /(p \varepsilon)
$$

the elastic moduli are given by:

$$
K_{p}(w)=\mu_{p} \exp \left[-p \gamma_{p} w\right]\left(2 \exp \left[-p \gamma_{p} w\right]-1\right), \quad 1 \leq p \leq 3 .
$$

Using (9) and (12), we can compute the strain dependence of the dimensionless parameters $\alpha(w)$ and $\beta(w)$. Depending on the choice of the microscopic parameters $\mu_{p}$ and $\gamma_{p}$ we obtain different paths in the $\alpha$ - $\beta$ plane; each path starts in the stable region and eventually reaches the instability threshold. If the potentials are identical for $\mathrm{NN}, \mathrm{NNN}$ and $\mathrm{NNNN}$ interactions $\left(\mu_{1}=\mu_{2}=\mu_{3}, \gamma_{1}=\gamma_{2}=\gamma_{3}\right)$, the resulting homogeneous equilibrium branch (path 1 in figure 1) crosses the macroinstability boundary $M N$, activating the instability mode $v_{n}=1$. The corresponding macroscopic stress-strain relation $\sigma(w)$ is shown in figure 3 . In this case the bifurcation point $A_{1}$ correspond to the failure of the LegendreHadamard conditions for the macroscopic energy $\left(\sigma^{\prime}(w)=0\right)$.

Suppose next that the three considered interactions (NN, NNN and NNNN) are governed by different Morse potentials. For instance, we may assume that $U_{1}^{\prime}(r)=U_{2}^{\prime}(r)$ but that the force associated with the NNNN interaction $U_{3}^{\prime}(r)$ decays at large $r$ slower than $U_{1}^{\prime}(r)$. If $\gamma_{3}$ is sufficiently smaller than $\gamma_{1}=\gamma_{2}$, the corresponding equilibrium branch (path 2 in figure 1) crosses the incommensurate microinstability boundary. The stability is lost at point $A_{2}$ where $\sigma^{\prime}(w)>0$, i.e. before the macroscopic threshold.

Now suppose that $U_{2}^{\prime}(r)$ decays slower than $U_{1}^{\prime}(r)$ but faster than $U_{3}^{\prime}(r)$ (e.g. $\gamma_{1}=1$, $\left.\gamma_{2}=0.4, \gamma_{3}=0.2\right)$. The corresponding equilibrium path 3 starts in the stable region where all three elastic moduli are positive (see figure 1 ). As $w$ increases, $K_{1}(w)$ approaches zero faster than the other two moduli, so that $\alpha$ and $\beta$ both tend to infinity. When $K_{1}$ changes sign, parameters $\alpha$ and $\beta$ become infinite and the path re-emerges in the stable region with $K_{1}<0$ (figure 2). The failure of the homogeneous state at $A_{3}$ takes place via incommensurate microinstability before the macroinstability threshold.

Finally, assume that NNN and NNNN interactions potentials are identical and that the corresponding forces decay slower than $U_{1}^{\prime}(r)\left(\gamma_{1}=1, \gamma_{2}=\gamma_{3}=0.4\right)$. Then we obtain path 4 which crosses the commensurate microinstability boundary at point $A_{4}$ to the right of point $N$ in figure 1 . The corresponding microinstability again precedes the macroinstability (see figure 3 ). 
(a)

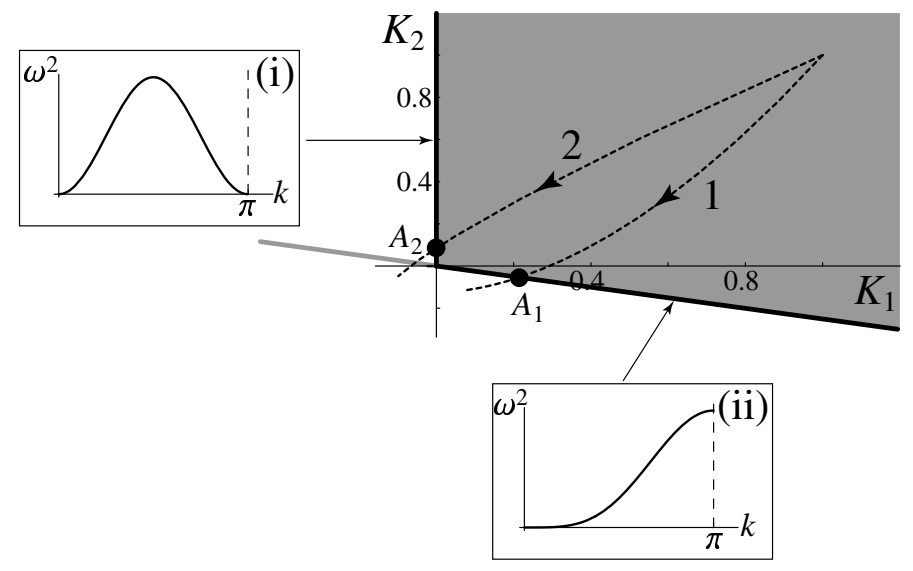

(b)

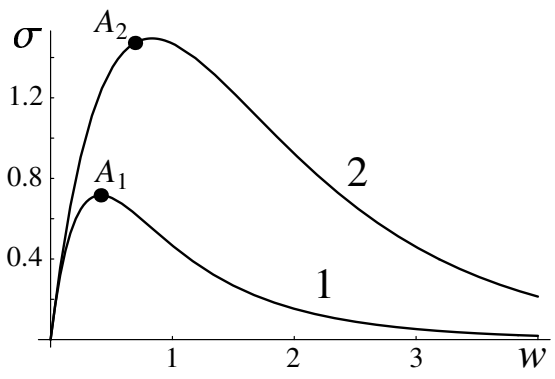

Figure 4: (a) Stability diagram for the discrete model with $q=2$. The trivial solution is stable in the gray area. Stability boundaries: (i) commensurate microinstability, $K_{1}=0$; (ii) macroinstability, $K_{1}+4 K_{2}=0$. In the classical continuum model the trivial solution is stable above the gray line $K_{1}+4 K_{2}=0$. The dotted curves show deformation paths: $\gamma_{2}=1$ (path 1) and $\gamma_{2}=0.4$ (path 2). In both cases $\mu_{1}=\mu_{2}=\gamma_{1}=1$. (b) The corresponding stress-strain curves.

In the special case when the only two interactions are NN and NNN $\left(q=2, K_{3}=0\right)$, the stability conditions (8) reduce to $K_{1}+4 K_{2}>0, K_{1}>0$ [15]; see the shaded region in figure $4 \mathrm{a}$. In this case the microinstability is necessarily commensurate, and it takes place at $K_{1}=0$. As before, the path with identical Morse potentials for NN and NNN interactions (path 1) leads to macroinstability. If $U_{2}^{\prime}(r)$ decays slower than $U_{1}^{\prime}(r)$, the instability becomes microscopic (see figure $4 \mathrm{~b}$ ).

\section{Quasicontinuum approximation}

A higher gradient quasicontinuum approximation of a lattice model is obtained by replacing the discrete dispersion relation (4) by the first few terms of its Taylor expansion around $k=0[16,17]$. To capture all three types of instabilities (long-wave, commensurate and incommensurate) exhibited by the discrete model with $q \geq 3$, it is necessary to consider a polynomial expansion of at least sixth order:

$$
\omega^{2}(k) \approx k^{2}\left(E+A_{1} k^{2}+A_{2} k^{4}\right),
$$


where the Taylor coefficients are

$$
\begin{aligned}
& E=K_{1}+4 K_{2}+9 K_{3} \\
& A_{1}=-\frac{K_{1}+16 K_{2}+81 K_{3}}{12} \\
& A_{2}=\frac{K_{1}+64 K_{2}+729 K_{3}}{360} .
\end{aligned}
$$

The quadratic part of the continuum energy function corresponding to (13) takes the form

$$
W=\frac{1}{2} \int_{-\infty}^{\infty}\left[E u_{x}^{2}+A_{1} \varepsilon^{2} u_{x x}^{2}+A_{2} \varepsilon^{4} u_{x x x}^{2}\right] d x
$$

To access stability of the homogeneous state in the continuum problem we need to solve the following eigenvalue problem:

$$
-\omega^{2} v=E v_{x x}-A_{1} \varepsilon^{2} v_{x x x x}+A_{2} \varepsilon^{4} v_{x x x x x x} .
$$

One can immediately see that if $A_{2}<0$ the energy is unbounded from below $\left(\omega^{2}(k)<0\right.$ for sufficiently large $|k|$ ). This short-wave instability is unphysical if the unstable wave length is shorter than the lattice spacing. To eliminate this possibility we can limit the class of perturbations by imposing a constraint $|k| \leq \pi$. This is achieved by replacing (13) with $\omega^{2}(k)=0$ outside the first Brillouin zone (for $|k|>\pi$ ). The resulting dispersion relation with a short wave cutoff is compared in figure 5 with the dispersion relation for the exact quasicontinuum model [18] obtained by replacing discrete dispersion by zero at $|k|>\pi$.

Despite its appearance, the proposed higher gradient approximation with a cutoff is essentially a continuum model with long-range spatial memory. Indeed, if we compute inverse Fourier transform of the truncated dispersion relation (13), we obtain the integral model

$$
W=\int_{-\infty}^{\infty} \int_{-\infty}^{\infty} \Phi(x-\xi) u(x, t) u(\xi, t) d x d \xi
$$

with the kernel

$$
\begin{aligned}
\Phi(x)= & \frac{1}{\pi x^{7}}\left[2 \pi x\left(\left(E+2 A_{1} \pi^{2}+3 A_{2} \pi^{4}\right) x^{4}-12\left(A_{1}+5 A_{2} \pi^{2}\right) x^{2}+360 A_{2}\right) \cos \pi x\right. \\
& +\left(\pi^{2} x^{6}\left(E+A_{1} \pi^{2}+A_{2} \pi^{4}\right)+2\left(E+6 A_{1} \pi^{2}+15 A_{2} \pi^{4}\right) x^{4}\right. \\
& \left.\left.+24\left(A_{1}+15 A_{2} \pi^{2}\right) x^{2}-720 A_{2}\right) \sin \pi x\right] .
\end{aligned}
$$

Meanwhile, the exact quasicontinuum model has the kernel (e.g. [18])

$$
\Phi_{D}(x)=\frac{4 \sin \pi x}{\pi x}\left(K_{1} \frac{x^{2}-1 / 2}{x^{2}-1}-\frac{2 K_{2}}{x^{2}-4}+K_{3} \frac{x^{2}-9 / 2}{x^{2}-9}\right)
$$



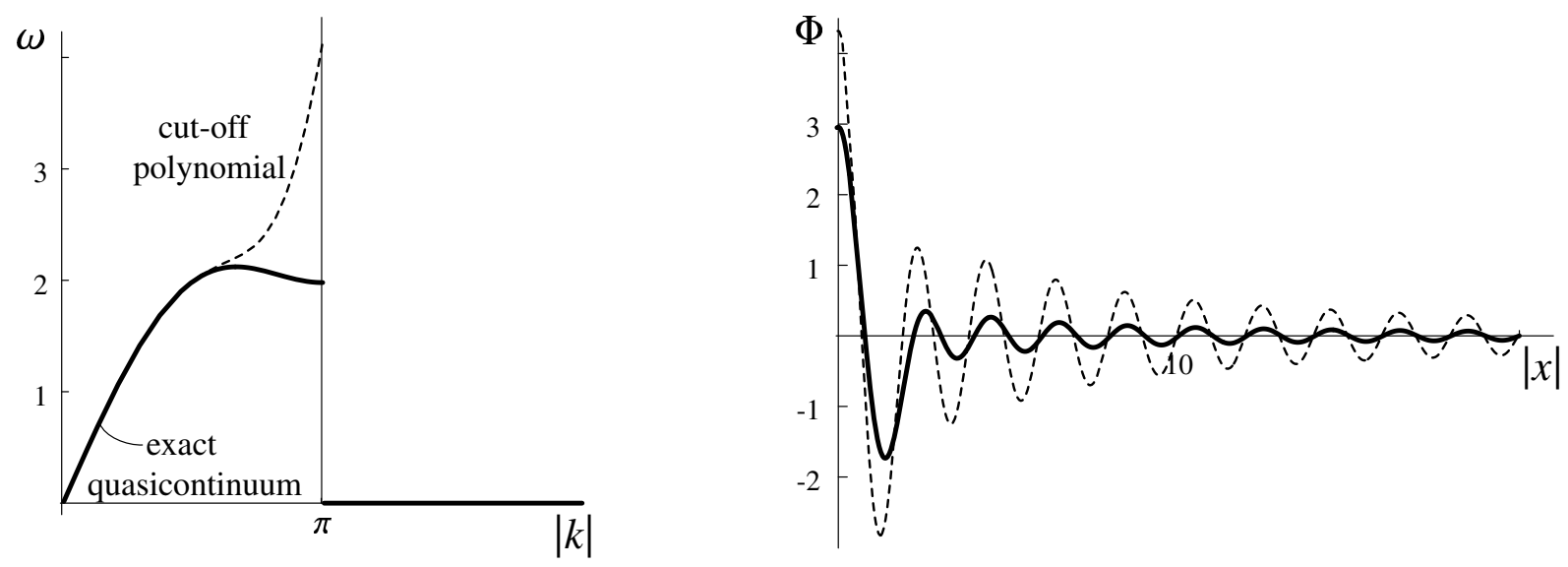

Figure 5: (a) Dispersion relations $\omega(k)$ for the exact quasicontinuum model (thick solid line) and the cutoff polynomial approximation (dashed). (b) The kernels $\Phi(x)$ of the exact quasicontinuum (solid) and cutoff polynomial (dashed) nonlocal models in physical space. Parameters: $K_{1}=1, K_{2}=0.5, K_{3}=-0.02$.

The two kernels are compared in figure 5b; one can see that the nonlocal interactions decay in both cases as a power of distance which is characteristic for the models with long-range interactions.

The proposed quasicontinuum model can be used to study stability of the homogeneous state. Following the same procedure as in the discrete case, we obtain the following necessary and sufficient conditions of stability:

$$
\begin{aligned}
& E>0 \\
& E+A_{1} \pi^{2}+A_{2} \pi^{4}>0 \\
& 4 A_{2} E-A_{1}^{2}>0 \text { if } 0<-\frac{A_{1}}{2 A_{2}}<\pi^{2} .
\end{aligned}
$$

The first condition, indicating macroinstability, coincides with $(8)_{1}$. The second condition is the analog of $(8)_{2}$, and the unstable mode is again $k=\pi$. The last inequality in (20) is analogous to $(8)_{3}$; its failure corresponds to incommensurate microinstability with the wave number

$$
k=k^{*}=\sqrt{-\frac{A_{1}}{2 A_{2}}} .
$$

A combined stability diagram illustrating conditions 20 is presented in figure 6 where it is compared with the stability diagram for the discrete model.

Observe first that in the discrete and quasicontinuum models at $K_{1}>0$ the stability boundaries are tangent at point $M$ where the unstable wave is infinitely long $(k=0)$. 
(a) $K_{1}>0$

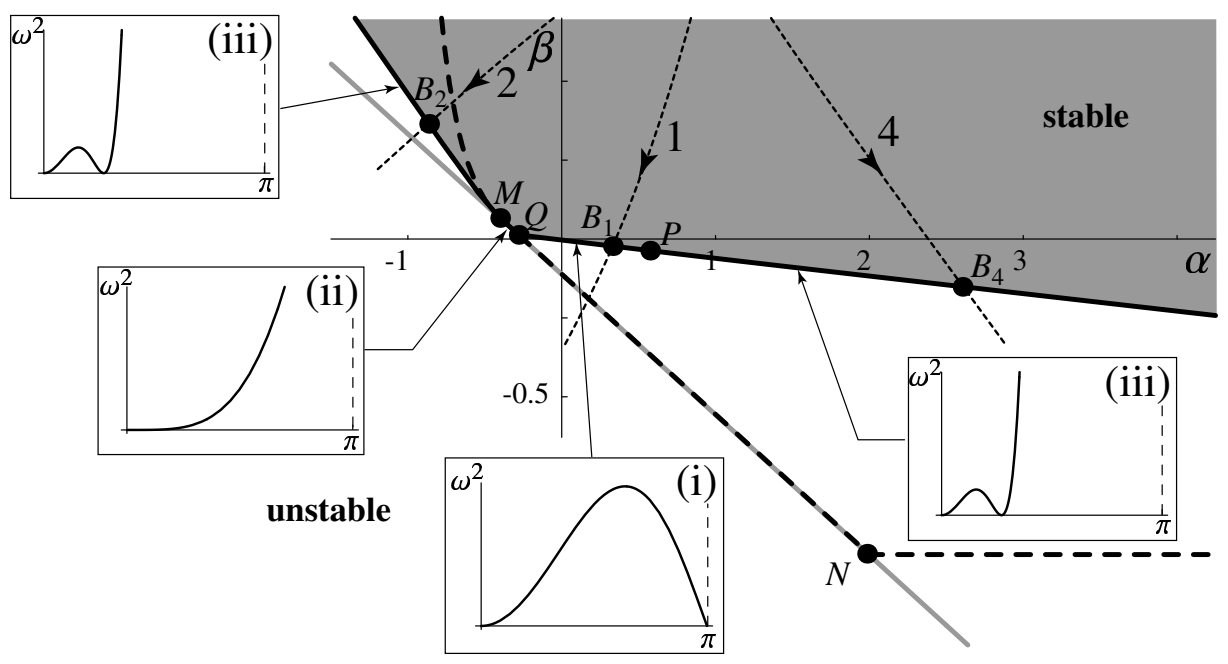

(b) $K_{1}<0$

(c)
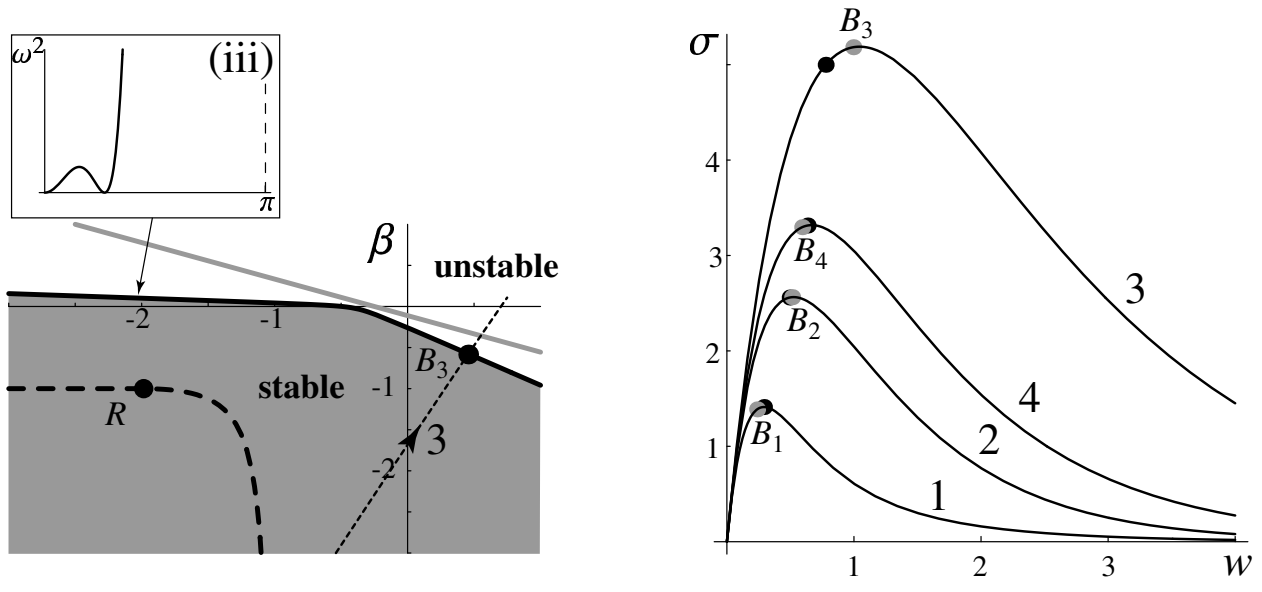

Figure 6: Stability diagram for the quasicontinuum polynomial model with the cutoff compared with the discrete stability diagram. Regions of stability are shown in gray, the corresponding graphs of $\omega^{2}(k)$ (dispersion relations) are shown in the inserts. Dashed curves indicate stability bounds for the discrete model. The two main diagrams correspond to the cases (a) $K_{1}>0$ and (b) $K_{1}<0$. Stability boundaries: (i) commensurate microinstability, $E+A_{1} \pi^{2}+A_{2} \pi^{4}=0$ $(P Q)$; (ii) macroinstability, $E=0(M Q)$; (iii) incommensurate microinstability, $4 A_{2} E-A_{1}^{2}=0$ (above $M$, below $P$ and entire boundary of $K_{1}<0$ stability region in (b)). Classical continuum model predicts stability above the gray line in (a) and below it in (b). In (c) we present stressstrain relations along the deformation paths (the same as in figure 1) shown by the dotted curves in (a) and (b), with bifurcation points for quasicontinuum model shown by gray circles and for the discrete model by black circles. 
Above point $M$ the quasicontinuum model reproduces the main qualitative features of the discrete diagram. Between points $M$ and $Q=\left(-4\left(\pi^{2}-3\right) /\left(13 \pi^{2}-30\right),\left(\pi^{2}-6\right) /\left(3\left(13 \pi^{2}-\right.\right.\right.$ $30)$ ) the instability mode is macroscopic in both models; this interval, however, is much shorter in the quasicontinuum model. Between points $Q$ and $P=\left(-\left(120-80 \pi^{2}+\right.\right.$ $\left.3 \pi^{4}\right) /\left(10\left(30-26 \pi^{2}+3 \pi^{4}\right)\right),-\left(30 \pi^{2}-\pi^{4}-90\right) /\left(45\left(30-26 \pi^{2}+3 \pi^{4}\right)\right)$ the stability loss is via microscopic mode with $k=\pi$. Below $P$ the instability mode is again incommensurate; such transition from commensurate to incommensurate instability is not observed in the discrete case. Overall, the quasicontinuum theory underestimates stability domains in the region $\left\{K_{1}>0, K_{2}>0, K_{3}<0\right\}$. This is due to the larger contribution of the oscillation-producing $K_{3}$ terms in the polynomial model compared to the discrete case.

At $K_{1}<0$ the quasicontinuum approximation has the opposite effect: it overestimates the stability of the homogeneous state. This is again caused by the very nature of the polynomial approximation: for positive $K_{2}$ and $K_{3}$ (which at $K_{1}<0$ result in negative $\alpha$ and $\beta$ ) the smoothening quadratic and quartic terms in the dispersion relation easily dominate the unstable contribution due to $K_{1}$. Notice also that while in the discrete case the boundary of the stable domain contains a segment corresponding to commensurate instability (which at point $R$ becomes incommensurate), in the polynomial quasicontinuum model the whole instability boundary is due to incommensurate mode.

It is instructive to compare the instability mechanisms along the four deformation paths considered earlier for the discrete model with the predictions of the quasicontinuum models (see figure 6). Along path 1 the quasicontinuum approximation predicts a commensurate microinstability (at point $B_{1}$ ), whereas the discrete model predicts macroscopic instability. Both models predict incommensurate instability along paths 2 and 3 , with stability failure somewhat delayed in the quasicontinuum model. Finally, path 4 is predicted to pass through microinstability boundary by both models; the mode of instability is, however, commensurate in the discrete case and incommensurate in the quasicontinuum approximation. The analysis of the relative position of the bifurcation points on the stress-strain curves, presented for both models in figure 6c, shows a reasonable quantitative agreement.

\section{Conclusions}

It has been long recognized that higher gradient approximations of lattice models generate in the continuum limit either unbounded or nonpositive definite operators leading to ill-posed mathematical problems (e.g. $[19,20]$ ). To overcome this difficulty we propose to regularize such operators by restricting them to a finite sphere in the Fourier space. At short wave lengths the resulting quasicontinuum model effectively replaces partial differential equations by integral equations. The dual nature of such cutoff polynomial models may be used to design hybrid computational schemes filtering parasitic small-scale oscillations while taking full advantage of the availability of simple partial differential equations 
for slowly varying fields. As we showed, the new quasicontinuum model combines the analytical simplicity of the gradient models at long waves with the physically correct description at small scales provided by models with long spatial memory. In particular, the approximation was shown to be sufficient to capture the whole spectrum of short-wave instabilities exhibited by the prototypical lattice model.

Acknowledgements. This work was supported by the NSF grants DMS-0102841 (L.T.) and DMS-0137634 (A.V.).

\section{References}

[1] M. Pitteri and G. Zanzotto, Continuum theories for phase transitions and twinning in crystals (Chapman and Hall, 2004).

[2] P. Toledano and V. Dmitriev, Reconstructive phase transitions: in crystals and quasicrystals (World Scientific, Singapore, 1996).

[3] T. Castan, A. Planes, and A. Saxena, Phys. Rev. B, 67, 134113 (2003).

[4] S. Kartha, J. Krumhansl, J. Sethna, and L. Wickham, Phys. Rev. B, 52, 803 (1995).

[5] X. Huang, C. Bungaro, V. Godlevsky, and K. M. Rabe, Phys. Rev. B, 65, 014108 (2002).

[6] D. C. Wallace, Thermodynamics of crystals (Dover Publications, 1998).

[7] N. Triantafyllidis and S. Bardenhagen, J. Mech. Phys. Solids, 44, 1891 (1996).

[8] T. Janssen and A. Janner, Adv. Phys., 36, 519 (1987).

[9] G. Fadda, L. Truskinovsky, and G. Zanzotto, Phys. Rev. B, 66, 174107 (2002).

[10] W. Cao, G. R. Barsch, Phys. Rev. B, 41, 4334 (1990).

[11] P. M. Chaikin, T. C. Lubensky, Principles of condensed matter physics (Cambridge University Press, 2000)

[12] B. Houchmandzadeh, J. Lajzerowicz, and E. Salje, J. Phys.-Condens. Mat., 4, 9779 (1992).

[13] T. Janssen and J. Tjon, Phys. Rev. B, 25, 3767 (1982).

[14] R. Hill, J. Mech. Phys. Solids, 10, 1 (1962). 
[15] L. Truskinovsky and A. Vainchtein, J. Mech. Phys. Solids, 52, 1421 (2004).

[16] G. R. Barsch and K. Krumhansl, Metall. Trans. A, 19, 761 (1988).

[17] R. D. Mindlin, Int. J. Solids Struct., 1, 417 (1965).

[18] I.A. Kunin, Elastic Media with Microstructure I: One-Dimensional Models, Vol. 26 of Solid-State Sciences (Springer-Verlag, Berlin-Heidelberg-New York, 1982).

[19] C. I. Christov, G. A. Maugin, and M. G. Velarde, Phys. Rev. E, 54, 3621 (1996).

[20] P. Rosenau, Phys. Let. A, 311, 39 (2003). 